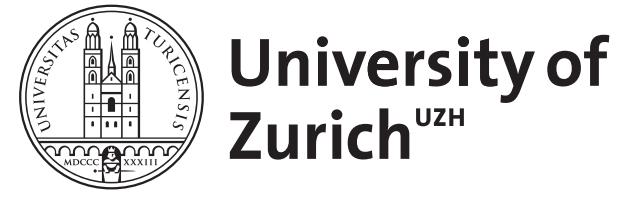

\title{
Model-based random forests for ordinal regression
}

\author{
Buri, Muriel ; Hothorn, Torsten
}

\begin{abstract}
We study and compare several variants of random forests tailored to prognostic models for ordinal outcomes. Models of the conditional odds function are employed to understand the various random forest flavours. Existing random forest variants for ordinal outcomes, such as Ordinal Forests and Conditional Inference Forests, are evaluated in the presence of a non-proportional odds impact of prognostic variables. We propose two novel random forest variants in the model-based transformation forest family, only one of which explicitly assumes proportional odds. These two novel transformation forests differ in the specification of the split procedures for the underlying ordinal trees. One of these split criteria is able to detect changes in non-proportional odds situations and the other one focuses on finding proportional-odds signals. We empirically evaluate the performance of the existing and proposed methods using a simulation study and illustrate the practical aspects of the procedures by a re-analysis of the respiratory sub-item in functional rating scales of patients suffering from Amyotrophic Lateral Sclerosis (ALS).
\end{abstract}

DOI: https://doi.org/10.1515/ijb-2019-0063

Posted at the Zurich Open Repository and Archive, University of Zurich ZORA URL: https://doi.org/10.5167/uzh-197565

Journal Article

Accepted Version

Originally published at:

Buri, Muriel; Hothorn, Torsten (2020). Model-based random forests for ordinal regression. International Journal of Biostatistics, 16(2):20190063.

DOI: https://doi.org/10.1515/ijb-2019-0063 


\section{Introduction}

Many disease stages or health conditions in clinical research are quantified by ordinal scores (Whegang, Basco, Gwét, and Thalabard, 2010, Roozenbeek, Lingsma, Perel, Edwards, Roberts, Murray, Maas, and Steyerberg, 2011, Tanadini, Steeves, Curt, and Hothorn, 2016, Peterson, Vock, Powers, Emery, Cruz, Hunsberger, Jain, Pett, Neaton, and Group, 2017, among many others). However, statistical methods commonly used for the analyses of ordinal score outcomes, most prominently approaches originally developed for continuous outcomes, seldom take into account the underlying structure of the outcome variable. As often pointed out (for example by Tanadini et al., 2016), methods for continuous outcomes ignore the fact that differences between adjacent categories of an ordinal outcome are not comparable and assumptions like variance homogeneity are inappropriate. Addressing these issues, Roozenbeek et al. (2011) emphasized the importance and added value of statistical methods tailored to ordinal outcomes in clinical trials. We follow this line of argumentation and propose two novel random forest variants explicitly tailored for the estimation of conditional distributions for ordinal outcomes. Existing random forest variants applicable to ordinal outcomes, namely Ordinal Forests (Hornung, 2019a) and Conditional Inference Forests (Hothorn, Hornik, and Zeileis, 2006), rely on a translation of ordinal measurements into artificial continuous scores which are subsequently analyzed as if they were continuous outcome variables. In contrast, the novel random forest variants proposed in this paper are based on suitable parametric models specifically tailored to ordinal data and do not rely on an underlying latent continuous variable.

Prognostic models have been playing an important role in medical research for a long time (Moons, Royston, Vergouwe, Grobbee, and Altman, 2009, Royston, Moons, Altman, and Vergouwe, 2009, Hemingway, Croft, Perel, Hayden, Abrams, Timmis, Briggs, Udumyan, Moons, Steyerberg, Roberts, Schroter, Altman, and Riley, 2013, Riley, Hayden, Steyerberg, Moons, Abrams, Kyzas, Malats, Briggs, Schroter, Altman et al., 2013, Steyerberg, Moons, van der Windt, Hayden, Perel, Schroter, Riley, Hemingway, Altman, Group et al., 2013, Hingorani, Windt, Riley, Abrams, Moons, Steyerberg, Schroter, Sauerbrei, Altman, and Hemingway, 2013, among many others). In addition to the task of predicting disease progression in patients, prognostic models can also be used as a reliable support to enroll adequate patients for therapeutic intervention trials. Recently, random forests have received strongly increasing attention in medical research as more researchers apply variants of this method for building prognostic (Hothorn and Jung, 2014, Beaulieu-Jones, Greene, and the Pooled Resource Open-Access ALS Clinical Trials, 2016, Ong, Tan, and Holbrook, 2017, Pfohl, Kim, Coan, and Mitchell, 2018) and predictive models (i.e. models featuring differential treatment effects, Seibold, Zeileis, and 
Hothorn, 2018).

On a more technical level, we introduce parametric model-based prognostic random forests for ordinal regression in the flexible framework of transformation forests (Hothorn and Zeileis, 2017). In Section 2, we define novel split criteria for prognostic model-based trees which are powerful in both the proportional and the non-proportional odds setting. In an artificial prognostic model setting introduced in Section 3 and discussed in Section 3.2, we empirically evaluate the performance of the two proposed variants for model-based random forests for ordinal regression in comparison to the recently proposed Ordinal Forest algorithm by Hornung (2019a) and the established Conditional Inference Forest algorithm (Hothorn et al., 2006). Conditional Inference Forests apply linear-by-linear association tests (Agresti, 2002) for split-point selection and thus essentially replace the ordered outcome categories by user-defined numeric scores (score-independent tests are discussed in Winell and Lindbäck, 2018). The Ordinal Forest algorithm by Hornung (2019a) also replaces the ordinal outcome by score values. The specific score values are optimized by maximizing the out-of-bag prediction performance of the classical regression forests by Breiman, Friedman, Olshen, and Stone (1984) (details are explained in Hornung, 2019a). This maximization procedure is conditional on a pre-defined performance function which is described in Hornung (2019a, Section 2.1.2.).

In Section 4, we investigate the practical aspects of the different procedures by comparing prognostic models fitted to data collected from several clinical trials for Amyotrophic Lateral Sclerosis (ALS). ALS is a devastating neurodegenerative disease which attacks the nerve cells responsible for sending instructions to the muscles lead to weakness, muscle waste, and paralysis. The data used were extracted from the Pooled Resource Open-Access ALS Clinical Trials (PRO-ACT, https://nctu.partners.org/ProACT) database (Atassi, Berry, Shui, Zach, Sherman, Sinani, Walker, Katsovskiy, Schoenfeld, Cudkowicz et al., 2014). ALS patients suffer rapid disease progression, hence, early identification of potential predictors of disease progression is crucial for taking meaningful clinical and therapeutic decisions to reduce the individuals risk (Riley et al., 2013). However, the identification of prognostic factors and the subsequent development of models forecasting disease progression of ALS is a long-standing and difficult, yet very important problem. The availability of such an instrument would, for example, allow the planning of more powerful clinical trials by means of efficient patient stratification (Chiò, Logroscino, Hardiman, Swingler, Mitchell, Beghi, and Traynor BG on behalf of the Eurals Consortium, 2009). Two approaches have been used in the past, namely the search for prognostic models for the overall survival time after diagnosis (Kimura, Fujimura, Ishida, Nakajima, Furutama, Uehara, Shinoda, Sugino, and Hanafusa, 2006, Zoccolella, Beghi, Palagano, Fraddosio, Guerra, 
Samarelli, Lepore, Simone, Lamberti, Serlenga, and Logroscino G for the SLAP Registry, 2008, Fujimura-Kiyono, Kimura, Ishida, Nakajima, Hosokawa, Sugino, and Hanafusa, 2011, Beaulieu-Jones et al., 2016, Mandrioli, Rosi, Fini, Fasano, Raggi, Fantuzzi, and Bedogni, 2017, Ong et al., 2017, Pfohl et al., 2018, among many others) and the prognosis of a functional assessment of patients via the ALSFRS (ALS functional rating scale; Brooks, Sanjak, Ringel, England, Brinkmann, Pestronk, Florence, Mitsumoto, Szirony, and Wittes, 1996) and ALSFRS-R scores (Cedarbaum, Stambler, Malta, Fuller, Hilt, Thurmond, and Nakanishi, 1999, Hothorn and Jung, 2014). Here, we focus on the prognosis of the respiratory sub-item which is an ordinal assessment of the patient's need for ventilatory support.

\section{Methods}

A prognostic model for an ordered outcome $Y \in \Xi=\left\{c_{1}<\cdots<c_{K}\right\}$ measured at $K$ possible categories describes the impact of prognostic variables $X \in \chi$ on the conditional distribution function $\mathbb{P}(Y \leq y \mid X=x)$. Without loss of generality, we can parameterise such a model as

$$
\mathbb{P}(Y \leq y \mid X=x)=\operatorname{expit}\left(a(y)^{\top} \vartheta(x)\right), \quad y \in\left\{c_{1}<\cdots<c_{K}\right\} .
$$

This formulation in terms of a transformation model allows the conditional logodds function $a(y)^{\top} \vartheta(x)$ to be defined by some basis function $a: \Xi \rightarrow \mathbb{R}^{K-1}$ and a conditional parameter function $\vartheta: \chi \rightarrow \mathbb{R}^{K-1}$. The latter function is typically estimated based on data from $i=1, \ldots, N$ independent subjects with prognostic variables $x_{i} \in \chi$ and an observed ordered outcome $Y=y_{i} \in \Xi$. A suitable basis function $a$ for $K$ ordered categories is the unit vector $e_{K-1}$ of length $K-1$, where the element $k$ corresponding to $y=c_{k}, 1 \leq k<K$, is one.

We shall use a specific version of transformation forests (Hothorn and Zeileis, 2017) to estimate $\hat{\vartheta}_{N}(x)$ and discuss the necessary adaptations for ordinal outcomes in the following. Transformation forests, and also more general random forests, can be understood as local adaptive likelihood estimators (Athey, Tibshirani, and Wager, 2019, Hothorn and Zeileis, 2017, Schlosser, Hothorn, Stauffer, and Zeileis, 2019). In our setup, we obtain an estimator for the conditional parameter function $\vartheta(x)$ evaluated for a patient with prognostic variables $x$ by means of a random forest-type estimator $\hat{\vartheta}_{N}(x)$, defined as the maximizer of the sum of the weighted $\log$-likelihood contributions $\ell_{i}(\vartheta), i=1, \ldots, N$ :

$$
\hat{\vartheta}_{N}(x)=\underset{\vartheta \in \mathbb{R}^{K-1}}{\arg \max } \sum_{i=1}^{N} w_{i}(x) \ell_{i}(\vartheta)
$$


for $\vartheta=\left(\vartheta_{1}, \ldots, \vartheta_{K-1}\right)^{\top} \in \mathbb{R}^{K-1}$ with $\vartheta_{1} \leq \vartheta_{2} \leq \cdots \leq \vartheta_{K-1}$. The nearest-neighbor weights $w_{i}(x)$ obtained from a given forest measure the similarity of the two conditional distribution functions $\mathbb{P}\left(Y \leq y \mid X=x_{i}\right)$ and $\mathbb{P}(Y \leq y \mid X=x)$ given a specific configuration $x$ of the prognostic variables. For cases for which $x_{i}$ is similar to $x$, the weight $w_{i}(x)$ is large, and thus the $i$ th observation is influential on $\hat{\vartheta}_{N}(x)$. More precisely, the nearest-neighbor weight $w_{i}(x)$ counts the number of times $x_{i}$ and $x$ are classified in the same terminal node by the single trees in the random forest. Hence, the weight is close to zero when $x_{i}$ and $x$ are elements of distinct terminal nodes for most trees in the forest. This method for tree aggregation has been around for some time (Hothorn, Lausen, Benner, and Radespiel-Tröger, 2004, Meinshausen, 2006, Lin and Jeon, 2006) but only recently lead to a more general understanding of random forests (Athey et al., 2019, Hothorn and Zeileis, 2017).

The log-likelihood contribution $\ell_{i}(\vartheta)$ of the $i$ th subject is obtained from the unconditional model

$$
\mathbb{P}(Y \leq y)=\operatorname{expit}\left(a(y)^{\top} \vartheta\right), \quad \vartheta=\left(\vartheta_{1}, \ldots, \vartheta_{K-1}\right)^{\top} \in \mathbb{R}^{K-1}
$$

An equivalent formulation of the log-odds function is $a(y)^{\top} \vartheta=\vartheta_{k}$ where $y=c_{k}$ for some $k=1, \ldots, K-1$. The latter terminology is used by default when describing cumulative models for ordinal outcomes but makes it hard to see the connections to other types of transformation models with generic transformation function $a(y)^{\top} \vartheta$, as for example discussed in Hothorn and Zeileis (2017) or Hothorn, Möst, and Bühlmann (2018).

Following Hothorn et al. (2018), the unconditional log-likelihood contributions are given by

$$
\ell_{i}(\vartheta)=\log \left(\mathbb{P}\left(Y=y_{i}\right)\right)= \begin{cases}-\log \left(1+\exp \left(-\vartheta_{1}\right)\right) & y_{i}=c_{1} \\ \log \left(\operatorname{expit}\left(\vartheta_{k}\right)-\operatorname{expit}\left(\vartheta_{k-1}\right)\right) & y_{i}=c_{k}, 1<k<K \\ \log \left(1-\operatorname{expit}\left(\vartheta_{K-1}\right)\right) & y_{i}=c_{K} .\end{cases}
$$

The specification of the conditional parameter function (1) does not evince a direct link between the nearest-neighbor weights $w_{i}(x)$, and thus the underlying random forest, and the log-likelihood contributions $\ell_{i}(\vartheta)$. However, good performance can be achieved by implementing split statistics which are sensitive to changes in the model parameters $\vartheta$ (Athey et al., 2019, Hothorn and Zeileis, 2017, Schlosser et al., 2019 ) and thus to changes in the log-likelihood contributions $\ell_{i}(\vartheta)$ for the $i$ th observation.

In the following, we introduce two novel split statistics. The core idea is to regress scores derived from the unconditional parametric model (2) on prognostic 
variables $x$. For the sake of simplicity, we only discuss splits in the root node. First, let us consider the unconditional ordered logistic regression model

$$
\mathbb{P}(Y \leq y)=\operatorname{expit}\left(a(y)^{\top} \vartheta+\alpha\right), \quad \alpha \equiv 0
$$

featuring an explicit intercept term $\alpha$. However, the transformation $a(y)^{\top} \vartheta$ already contains an implicit intercept term, hence, we constrain $\alpha$ to zero. The corresponding $\log$-likelihood contributions are $\ell_{i}(\vartheta, \alpha)$ and the score contribution $s_{i}^{\alpha}(\vartheta)$ for observation $i$ is the partial derivative of the log-likelihood with respect to the intercept parameter $\alpha$

$$
s_{i}^{\alpha}(\vartheta)=\left.\frac{\partial \ell_{i}(\vartheta, \alpha)}{\partial \alpha}\right|_{\alpha=0} \in \mathbb{R} .
$$

A regression model explaining these scores by one or more of the prognostic variables $x$ assumes a deviation of a conditional model from the unconditional model which can be described as $\alpha(x) \neq 0$. This idea underlies many statistical learning procedures, such as gradient boosting (a boosting algorithm for ordinal outcomes using exactly these scores was proposed by Schmid, Hothorn, Maloney, Weller, and Potapov, 2011). For continuous outcomes $Y \in \mathbb{R}$, the scores with respect to $\alpha$ in model (3) are called Wilcoxon scores, although the term usually refers to the application of the empirical cumulative distribution function for the unconditional distribution in the model with $a\left(y_{i}\right)^{\top} \vartheta=\operatorname{logit}\left((N+1)^{-1} \cdot \operatorname{rank}\left(y_{i}\right)\right)$. Models explaining the scores $s_{i}^{\alpha}(\vartheta)$ by prognostic variables $x$ are powerful in detecting proportional odds deviations from model (3) of the form $\alpha(x) \neq \alpha=0$. Note that the score contributions $s_{i}^{\alpha}(\vartheta)$ do not carry much information in a non-proportional odds setting, i.e. when $\alpha(x) \equiv 0$ and $\vartheta(x) \neq \vartheta=$ const. Hereafter, we refer to transformation forests relying on the score contributions (4) for computing split statistics as $\operatorname{OTF}(\alpha)$, short for "Ordinal Transformation Forests assuming proportional odds".

For the specific case of non-proportional odds, the application of split statistics sensitive to changes in the parameters $\vartheta(x)$ is necessary. Consequently, we obtain score contributions directly from the unconditional model (2), more precisely as

$$
s_{i}(\vartheta)=\frac{\partial \ell_{i}(\vartheta)}{\partial \vartheta} \in \mathbb{R}^{K-1}
$$

Deviations from the unconditional model of the form $\vartheta(x) \neq \vartheta=$ const can thus be detected by models regressing these scores on $x$. Appropriate split-statistics for these multivariate scores are defined by Hothorn and Zeileis (2017) and the corresponding split algorithm is explained in Seibold et al. (2018, Appendix 2). We 
hereafter refer to the corresponding transformation forests based on score contributions (5) as "Ordinal Transformation Forests for non-proportional odds", in short $\operatorname{OTF}(\vartheta)$.

The primary aim of this paper is to compare random forest variants for ordinal outcomes, namely Ordinal Forests, Conditional Inference Forests, $\operatorname{OTF}(\alpha)$, and $\operatorname{OTF}(\vartheta)$ under proportional odds and lack thereof. We evaluate the prognostic performance of all competitors by means of the out-of-sample log-likelihood of $\tilde{N}$ additional validation subjects $\left(y_{l}, x_{l}\right), \imath=N+1, \ldots, N+\tilde{N}$ :

$$
\ell(N, \tilde{N})=\sum_{\imath=N+1}^{N+\tilde{N}} \log \left(\hat{\mathbb{P}}_{N}\left(Y=y_{\imath} \mid X=x_{\imath}\right)\right) .
$$

The application of this proper scoring rule helps to assess how generalizable the forests are to an independent data set.

It should be noted, however, that the novel OTFs implicitly optimise the likelihood via (1) whereas Conditional Inference Forests and Ordinal Forests do not have a notion of this performance measure. Like OTFs, Conditional Inference Forests can be used to compute conditional probability estimates and we use these to compute $\ell(N, \tilde{N})$. In contrast, the Ordinal Forest algorithm by Hornung (2019a) was designed to focus on predictions of the class values $\hat{Y}_{l}=c_{k}$ and not conditional probability estimates and thus a direct evaluation of $\ell(N, \tilde{N})$ is not possible. We dealt with this issue in two ways. For the comparisons in Section 3, we obtained conditional class probability estimates from Ordinal Forests using the classfreqtree list object of the predict.ordfor () function output. The matrix classfreqtree is of dimension $\tilde{N} \times K$, where the value in the $k$-th column of the $i$-th row contains the proportion of the trees in the forest that predicted class $c_{k}$ for observation $i$. This procedure allows us to calculate the out-of-bag log-likelihood, however, the comparison is strictly speaking not fair based on the different objectives of OTFs and Ordinal Forest algorithms. Therefore, we applied the Kullback-Leibler divergence as a means to directly compare a predicted class $\hat{Y}_{l}=c_{k}$ obtained from any of the forest variants to the true conditional distribution in our simulation experiments. The results of the latter performance measure are presented in Appendix A.2. The Kullback-Leibler divergence $\mathrm{KL}_{2}$ applied in the appendix allows a fair comparison of OTFs and Ordinal Forests.

\section{Empirical Evaluation}

We were interested in a comparison of Ordinal Forests, Conditional Inference Forests $(\mathrm{CForest}), \operatorname{OTF}(\alpha)$, and $\operatorname{OTF}(\vartheta)$ under proportional odds and the lack thereof for 
assessing the following hypotheses: (a) All methods perform similarly under proportional odds. (b) The $\mathrm{OTF}(\vartheta)$ forest employing a more general split statistic performs better under non-proportional odds. Our experiments utilized open-source implementations of these methods available in the R system for statistical computing (R Core Team, 2020). Ordinal Forests were fitted with the ordfor () function from the add-on package ordinalForest (Hornung, 2019b), CForest with the cforest () function from the party add-on package (Hothorn, Hornik, Strobl, and Zeileis, 2019), and the OTF variants with the function traforest () from the addon package trtf (Hothorn, 2019b).

As previously mentioned, the Ordinal Forest algorithm by Hornung (2019a) uses optimized score values instead of the class values for the ordinal outcome. The optimization of these score values is done by maximizing the out-of-bag prediction performance of the estimated regression forests. For the following comparisons, we distinguish between two different options for the quantification of the highest out-of-bag prediction performance. More precisely, these two options differ with respect to the choice of the version used for the so-called performance function that is inherent to the Ordinal Forest algorithm. The goal of the option equal is to obtain an Ordinal Forest so that the observations from each outcome category are classified with the same accuracy. In contrast, the option proportional has the goal of correctly classifying as many observations as possible, e.g. the prediction accuracy should be proportional to the sizes of the outcome categories. A detailed overview of the Ordinal Forest algorithm as well as the underlying performance functions is given in Hornung (2019a, Section 2.1.2).

\subsection{Data Generating Processes}

We simulated ordered outcomes $Y$ from a transformation model with conditional distribution function

$$
\mathbb{P}(Y \leq y \mid X=x)=\operatorname{expit}\left\{\xi(x) \cdot a(y)^{\top} \vartheta(x)+\alpha(x)\right\}
$$

with $y \in\left\{c_{1}<\cdots<c_{K}\right\}$ and $\vartheta=\left(\vartheta_{1}, \ldots, \vartheta_{K-1}\right)^{\top} \in \mathbb{R}^{K-1}$ featuring conditional parameter functions $\xi$ (scale term) and $\alpha$ (shift term) as functions of prognostic variables $x$. We defined $K=4$ ordinal categories for the outcome variable $Y$ with $\vartheta=\left(\vartheta_{1}, \ldots, \vartheta_{3}\right)^{\top}=\operatorname{logit}(.15, .5, .85)^{\top}$, implying unequal outcome category probabilities for $\mathbb{P}(Y \leq y \mid x)$ when $\xi(x) \equiv 1$ and $\alpha(x) \equiv 0$. We obtain data under proportional odds when choosing $\xi(x) \equiv 1$. Then, the log-odds ratio is $\alpha(x)$ and the data generating model (6) is equivalent to a nonlinear (in $\alpha(x)$ ) proportional odds logistic regression model. Non-proportional odds are obtained when $\xi(x) \neq 1$, regardless of the choice of $\alpha(x)$. 
Scale and shift conditional parameter functions $\xi(x)$ and $\alpha(x)$ were modeled using the function

$$
\mathrm{F}\left(x_{1}, x_{2}, x_{3}, x_{4}, x_{5}\right)=10 \sin \left(\pi x_{1} x_{2}\right)+20\left(x_{3}-.5\right)^{2}+10 x_{4}+5 x_{5}
$$

suggested by Friedman (1991). The output of $F$ was scaled to the $[-2.5,2.5]$ interval, denoted below as $\mathrm{F}^{\star}$. This choice restricted the odds ratios $\exp (\alpha(x))=$ $\exp \left(\mathrm{F}^{\star}\right)$ to values between $\exp (-2.5)=.08$ and $\exp (2.5)=12.18$. Four types of effects were simulated:

- No: no effect, without a scale term $\xi(x) \equiv 1$ and without shift $\alpha(x) \equiv 0$

- PO: proportional odds effect, without a scale term $\xi(x) \equiv 1$ but with shift $\alpha(x) \neq 0$

- Non-PO: non-proportional odds effect, with scale $\xi(x) \neq 1$ but without shift $\alpha(x) \equiv 0$

- Combined: a combination of "PO" and "Non-PO", with a scale term $\xi(x) \neq 1$ and with shift $\alpha(x) \neq 0$.

The effects in reference to model (6) can be summarized as follows:

\begin{tabular}{lll}
\hline & $\xi(x)$ & $\alpha(x)$ \\
\hline No & 1 & 0 \\
PO & 1 & $\mathrm{~F}^{\star}\left(x_{6}, \ldots, x_{10}\right)$ \\
Non-PO & $\exp \left(\mathrm{F}^{\star}\left(x_{1}, \ldots, x_{5}\right)\right)$ & 0 \\
Combined & $\exp \left(\mathrm{F}^{\star}\left(x_{1}, \ldots, x_{5}\right)\right)$ & $\mathrm{F}^{\star}\left(x_{6}, \ldots, x_{10}\right)$ \\
\hline
\end{tabular}

Additionally, we distinguish between low- and high-dimensional data. The lowdimensional prognostic variables were modeled with $J=15$ (10 prognostic and five additional noise) independent uniform variables, i.e. $X=\left(X_{1}, \ldots X_{J}\right) \sim U[0,1]^{J}$. The high-dimensional prognostic variables were modeled in the same manner, but with $J=60$ (10 prognostic and 50 additional noise) independent uniform variables.

All models were evaluated with respect to the out-of-sample log-likelihood difference $\ell(N, \tilde{N})-\ell(\xi, \alpha)$. The latter termrefers to the log-likelihood evaluated for the true parameters $\xi(x)$ and $\alpha(x)$ for validation subjects $\imath=N+1, \ldots, N+\tilde{N}$.

We prespecified a common set of hyperparameters for all random forests method to be compared: 250 trees (ntree) of maximal depth 10 (nodedepth) and not less than 20 observations in a terminal node (minbucket). For the lowdimensional setup, bagging was applied. Hence, all mtry $=J=15$ variables were used for splitting in a non-terminal node. In the high-dimensional setting, a restricted amount of input variables randomly sampled as candidates at each node for the random forest like algorithms was used; more precisely, mtry was set equal to the square root of the total numbers of explanatory variables $\sqrt{J}=\sqrt{60}$. Additional method-specific parameter tuning was not applied. Beside these introduced common hyperparameters, the function's default parameters were used for 
the simulation based empirical evaluation, except for the performance function perffunction of the Ordinal Forests. The Ordinal Forest specific default parameter values are nsets $=1000$, ntreeperdiv $=100$, nbest $=10$.

Note that all forests were grown with the same learning sub-samples of the original data. No form of early stopping was applied. We repeated each of the eight simulation scenarios (four effect types for low and high dimensions each) 100 times with learning and validation samples of size $N=250$ and $\tilde{N}=500$, respectively.

\subsection{Results}

The boxplots in Figure 1 show the distribution of the out-of-sample log-likelihood differences $\ell(N, \tilde{N})-\ell(\xi, \alpha)$. The two columns "Low" and "High" in Figure 1 distinguish between the low (five additional noise variables) and high (50 additional noise variables) dimensional data. The rows in Figure 1 distinguish between the four specific effect types (absent effect "No", proportional odds "PO", nonproportional odds "Non-PO", or a combination of PO and Non-PO "Combined"). Note that good performance of a specific random forest variant results in relatively high out-of-sample log-likelihood differences. Hence, larger out-of-sample loglikelihood differences are preferable. Figures showing the absolute log-likelihood results can be found in Appendix A.1. Additionally, we also provide the results of a sensitivity analysis for which the number of trees per forest was increased to ntree $=2000$ in Appendix A.1. It should be noted, however, that the number of trees is not a tuning parameter but using larger values provides a better approximation of the nearest neighbor weights. Larger forests of 2000 trees did not lead to substantial improvement compared to forests of 250 trees in our simulations, where the differences in results between these two settings are slightly larger in the case of Ordinal Forests. In the absence of any effect (first row of Figure 1), the random forest methods CForest, $\operatorname{OTF}(\alpha)$, and $\operatorname{OTF}(\vartheta)$ roughly performed equally well. Note that regardless of the score contributions used for splitting, the two competitors of the ordinal transformation forests $\operatorname{OTF}(\alpha)$ and $\operatorname{OTF}(\vartheta)$ achieved very similar results.

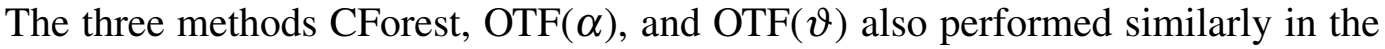
high dimensional simulation setting (first row, second column of Figure 1). Ordinal Forests performed less well in these situations, especially in low rather than in high dimensions.

As expected, the Ordinal Transformation Forest $\mathrm{OTF}(\alpha)$ explicitly designed for being powerful in detecting proportional odds deviations retrieved good results in the simulation setting where proportional odds were induced (second row of Figure 1). $\operatorname{OTF}(\alpha)$ and CForest performed also very similar in the other three settings. The more general $\operatorname{OTF}(\vartheta)$ performed less well, which might be explained by the 


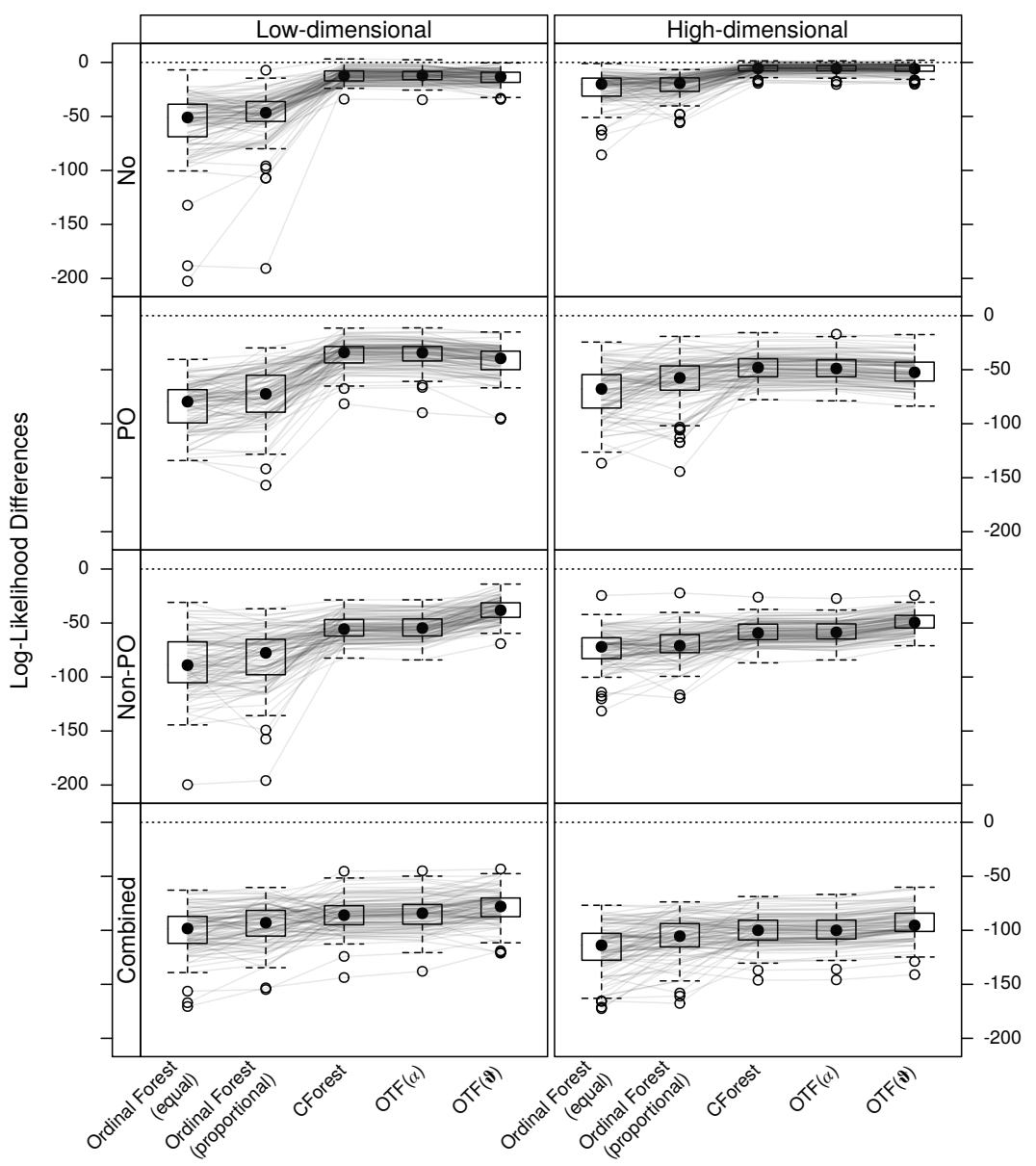

Figure 1

fact that, in the case of $\operatorname{OTF}(\vartheta)$, three parameters depend jointly on $x$ whereas in $\operatorname{OTF}(\alpha)$ only the scalar shift term $\alpha(x)$ had to be modeled.

The third and the fourth row of Figure 1 reflect the simulation settings for which scale and shift parameters had been intentionally introduced in the simulation setting. Hence, non-proportional odds ("non-PO") as well as a combination of scale and shift effects ("Combined") characterise these settings. The superior performance of the Ordinal Transformation Forest $\operatorname{OTF}(\vartheta)$ was in line with our hypothesis (b), as this method outperformed its competitors in the presence of nonconstant scale terms $\xi(x)$ (bottom four panels in Figure 1).

A comparison with respect to the Kullback-Leibler divergence (KL) as an 
additional performance measure can be found in Appendix A.2, again for forests of small and large number of trees. The results were very similar to the likelihoodbased assessment described in this section.

\section{ALS Venilatory Support}

The Pooled Resource Open-Access ALS Clinical Trials (PRO-ACT, https : / nctu . partners.org/ProACT) database contains longitudinal data of ALS patients who participated in one of 16 phase II and III trials and one observational study. The non-profit organization Prize4Life (http://www.prize4life.org/) initiated this project to enhance knowledge about ALS (Küffner, Zach, Norel, Hawe, Schoenfeld, Wang, Li, Fang, Mackey, Hardiman, Cudkowicz, Sherman, Ertaylan, GrosseWentrup, Hothorn, van Ligtenberg, Macke, Meyer, Schölkopf, Tran, Vaughan, Stolovitzky, and Leitner, 2015). The PRO-ACT database contains information on a broad variety of patient characteristics, such as vital signs, the patient's and family's history, and treatment information. Note that identification criteria, such as study centres, are not included in the database.

We focus on disease progression quantified by the revised ALSFRS (Cedarbaum et al., 1999). This scale is used by physicians to diagnose patients and assess their disease progression but also by researchers for selecting clinical trial participants as well as to measure the potential treatment effects. The scale consists of twelve items rating a patient's ability to perform common daily tasks, i.e. climbing stairs, writing, dressing, cutting food or handling cutlery. Each of the items is then scored with a five-point scale ranging from 0 to 4 , where 0 is "can't do" and 4 refers to "normal ability". The extracted data set with 2534 observations from the PROACT database contains the ALSFRS-R individual scores at baseline and half a year after diagnosis as well as baseline patient characteristics. The original data set consisted of 82 variables. A detailed description of patient characteristics is available from Seibold et al. (2018). Here, we report results of a complete case analysis for the prognosis of the individual ALSFRS-R item quantifying the need for ventilatory support ("Respiratory" with scores " 0 ": Invasive mechanical ventilation by intubation or tracheostomy < "1": Continuous use of BiPAP (Biphasic Positive Airway Pressure) during the night and day $<$ "2": Continuous use of BiPAP during the night $<$ “3": Intermittent use of BiPAP < "4": None) half a year after diagnosis. Consequently, the prognostic models only take into account the baseline ALSFRS-R item measurements immediately after diagnosis and the individual patient characteristics. This setup led to a final data set with 1013 observations and 70 explanatory variables. The ALSFRS-R scores for the "Respiratory" item defined the five-point ordinal outcome variable for different prognostic models. The absoulte numbers of 
patients per outcome category of the target variable "Respiratory" were 1 (category 0), 24 (category 1), 60 (category 2), 322 (category 3), and 606 (category 4).

The previously discussed OTF approaches along with unconditional and prognostic proportional odds logistic regression (Polr) models as well as CForest were applied; the following list gives an overview of all competitors:

- Polr(): unconditional proportional odds logistic regression (Polr) model, ignoring patient characteristics.

- $\operatorname{Polr}(\alpha)$ : prognostic proportional odds logistic regression (Polr) model with linear predictor $\alpha(x)=x^{\top} \beta$.

- CForest: prognostic conditional inference forest.

- $\operatorname{OTF}(\alpha)$ : prognostic ordinal transformation forest able to detect changes in proportional odds.

- $\operatorname{OTF}(\vartheta)$ : prognostic ordinal transformation forest able to detect changes in non-proportional odds.

The variants of the Ordinal Forest by Hornung (2019a) are not considered here because a fair comparison by means of the out-of-sample log-likelihood is not possible for the reasons discussed in Section 2.

To estimate the performance of the different procedures, we generated 100 random splits of the above introduced data set dividing it into learning and validation samples in proportion $3: 1$. The out-of-sample log-likelihood served as the overall performance measure to compare the competing methods to the observed score. Again, larger values reflecting a good performance are preferable. All random forest variants used the same hyperparameter settings as defined for the simulation study above. The number of input variables randomly sampled as candidates at each node for the random forest-like algorithms are set equal to the square root of the total numbers of explanatory variables mtry $=\sqrt{J}=\sqrt{70}$. $*$

Figure 2 shows the out-of-sample log-likelihood for the five different prognostic methods, each applied to the 100 random splits of the ALSFRS-R data set. Unsurprisingly, every method which takes the baseline patient characteristic variables into account outperformed the unconditional proportional odds logistic regression (Polr) model. Hence, one can state that it is beneficial to take available baseline measurements into account for potential prognostic models. The CForest as well as the ordinal transformation forests showed very similar results. The fact that $\operatorname{OTF}(\alpha)$ and $\operatorname{OTF}(\vartheta)$ performed very similarly allows one to conclude that nonproportional odds effects are negligible in prognostic models of the "Respiratory" sub-item half a year after diagnosis. This indicates that the finding from our simulation study, where $\operatorname{OTF}(\alpha)$ and $\operatorname{OTF}(\vartheta)$ also performed very similarly in cases where non-proportional odds were not necessary to capture the signal in the data, is appropriate. 


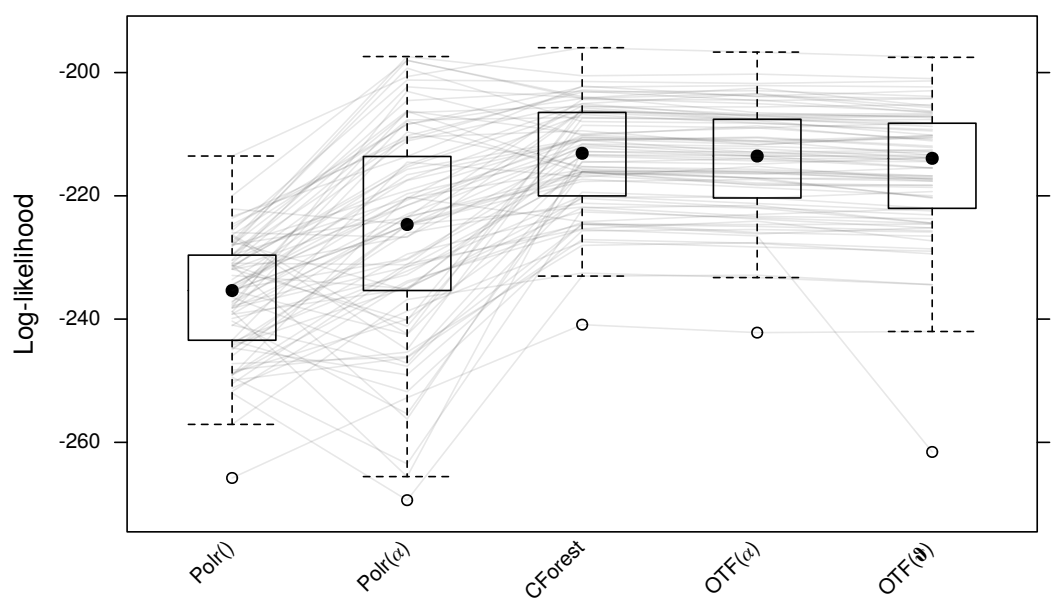

Figure 2

Overall, the log-likelihood distributions of the $\operatorname{Polr}(\alpha)$ model reveals relatively high variances in comparison to the other competitors. The superior performance of the random forest variants compared to the linear model $\operatorname{Polr}(\alpha)$ may be explained by a presumably non-linear impact of the prognostic variables $x$ or the presence of higher-order interaction effects.

One referee report posed the question how Ordinal Forests (proportional) compare to ordinal transformation forests allowing for non-proportional odds, $\operatorname{OTF}(\vartheta)$, in terms of the predicted scores. One can understand the two methods as two raters, assigning one of the categories $0, \ldots, 4$ to each ALS patient. For each of the 100 repetitions, we implemented the quadratic weighted Cohen's Kappa (Cohen, 1968) to assess the inter-rater reliability on the validation samples. Figure 3 indicates a rather high level of agreement for most validation samples.

\section{Discussion and Outlook}

Models for discrete conditional distribution functions are a natural choice for the analysis of ordinal outcomes. The linear proportional odds logistic regression model (McCullagh, 1980, Agresti, 2010, Tutz, 2011)

$$
\mathbb{P}\left(Y \leq c_{k} \mid x=X\right)=\operatorname{expit}\left(\vartheta_{k}+x^{\top} \beta\right), \quad \vartheta_{1} \leq \vartheta_{2} \leq \cdots \leq \vartheta_{K-1}
$$

respects the measurement properties of the ordinal outcome through ordered threshold parameters $\vartheta_{k}$. Our contribution of the novel random forest variant $\operatorname{OTF}(\alpha)$ 


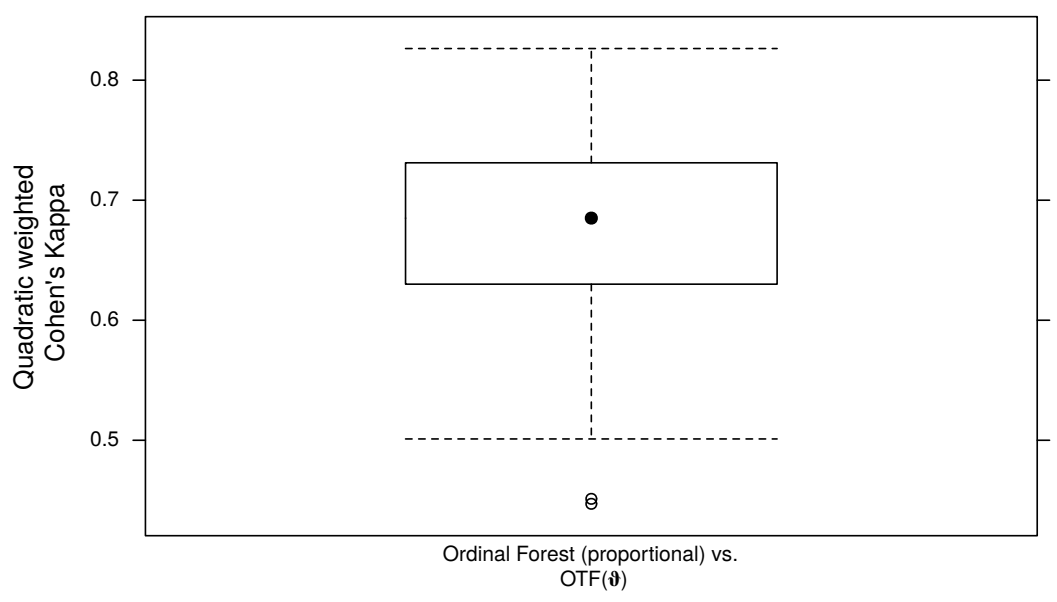

Figure 3

essentially replaces the linear predictor in this model with a flexible function of the prognostic variables $x$ while maintaining the proportional odds assumption. The second novel random forest variant, $\operatorname{OTF}(\vartheta)$, relaxes this restriction and allows the threshold parameters to depend on prognostic variables $\vartheta_{k}(x)$ directly. Because the models are still fully parametric, maximum likelihood estimation can be performed and the resulting random forest estimates are interpretable as maximizers of a local adaptive log-likelihood function in a rather simple model.

The empirical experiments reported here suggest that this combination of classical parametric models with novel machine learning technologies is beneficial, both in terms of prognostic accuracy as well as resulting model interpretability. We found that prognostic models for an ALS patient's need for ventilatory support do not require non-proportional odds to be taken into account. Thus, instead of comparing two predictive distributions of the ventilatory support outcome for two patients $i$ and $j$ with explanatory variables $x_{i}$ and $x_{j}$, a comparison between these two patients can be obtained and communicated in terms of the log-odds ratio $\hat{\alpha}\left(x_{i}\right)-\hat{\alpha}\left(x_{j}\right)$ independent of any specific category $k$ (cf. Equation 6).

The obtained results of the eight simulation settings, as well as the application to the ALSFRS data set, suggest that ordinal transformation forests outperform competing procedures. However, for making definitive conclusions on the prediction performance differences between the compared random forest methods, a larger-scale comparison study would be necessary as part of future research.

The methods presented here are closely related to models commonly used in 
survival analysis. Replacing the logit with the complementary log-log link function leads to the Cox model (Doksum and Gasko, 1990), where the proportional odds assumption translates into proportional hazards. Novel survival forest variants were proposed by Korepanova, Seibold, Steffen, and Hothorn (2019) in the same theoretical and computational framework of transformation forests (Hothorn and Zeileis, 2017).

The model-based approach to random forests allows for interesting extensions, for example the enrichment of models (2) or (3) with additional parameters, e.g. an explicit treatment effect. The aim would then be to identify differential treatment effects from randomized clinical trial data. The result is a predictive model featuring an explicit treatment effect $\beta$ that captures changes in the conditional distribution function induced by a specific treatment $(z=0$ for placebo and $z=1$ for treatment). In the simplest situation, model (3) extended by $\beta$ would result in

$$
\mathbb{P}(Y \leq y \mid Z=z)=\operatorname{expit}\left(a(y)^{\top} \vartheta+\alpha+\beta \mathbb{1}(z=1)\right), \quad \alpha=0 .
$$

The corresponding bivariate score contributions are then defined as

$$
s_{i}^{\alpha, \beta}(\vartheta)=\left.\frac{\partial \ell_{i}(\vartheta, \alpha, \beta)}{\partial(\alpha, \beta)^{\top}}\right|_{\alpha=0}=s_{i}^{\alpha}(\vartheta)(1, \mathbb{1}(z=1)) \in \mathbb{R}^{2} .
$$

Such models have been investigated for continuous and survival outcomes by Seibold et al. (2018) and Korepanova et al. (2019) and can also be applied when differential treatment effects for ordinal outcomes are of special interest.

\section{Computational Details}

All computations were performed using $\mathrm{R}$ version 3.6.3 (R Core Team, 2020). The code for data preprocessing of the PRO-ACT data is available in the TH.data addon package (Hothorn, 2019a). Patient-level data is available to registered users from https://nctu.partners.org/ProACT. OTF models were computed using the traforest () function from the trtf add-on package (Hothorn, 2019b). The trtf package was built on top of the infrastructure packages partykit (Hothorn and Zeileis, 2015) and mlt (Hothorn, 2020). Ordinal Forests were obtained from the ordinalForest add-on package (Hornung, 2019b). The party package (Hothorn et al., 2019) was used to compute Conditional Inference Forests (CForest). The code necessary to reproduce the empirical results reported in this paper is available from within $\mathrm{R}$ 
install.packages ("trtf", repos = "http://R-forge.R-project.org")

system.file("ordinal_forests", package = "trtf")

As a hands-on example, we demonstrate how the prognostic ordinal transformation forest $\operatorname{OTF}(\vartheta)$ allowing for non-proportional odds can be applied to the publically available hearth data set from the ordinalForest add-on package (Hornung, 2019b):

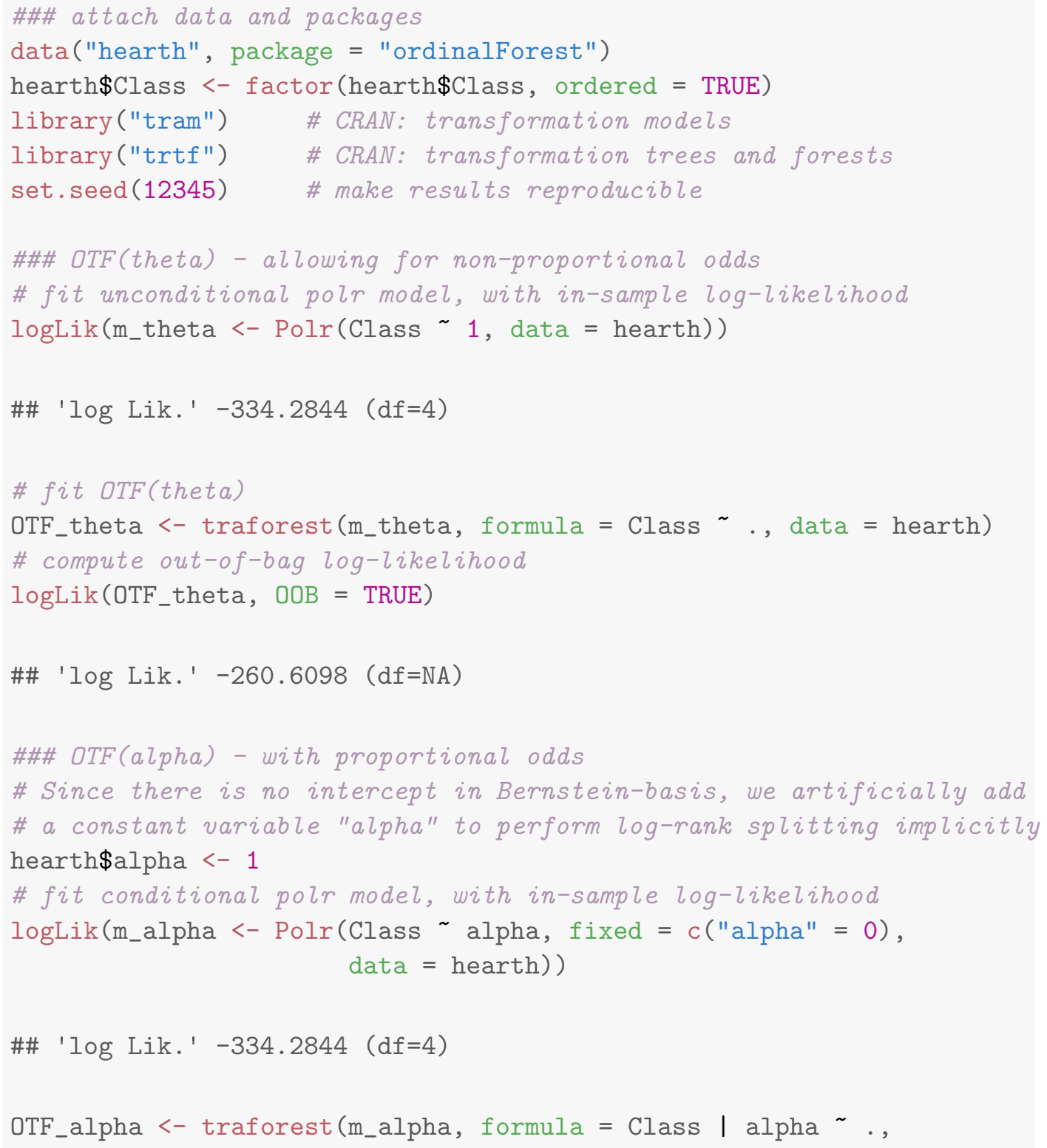




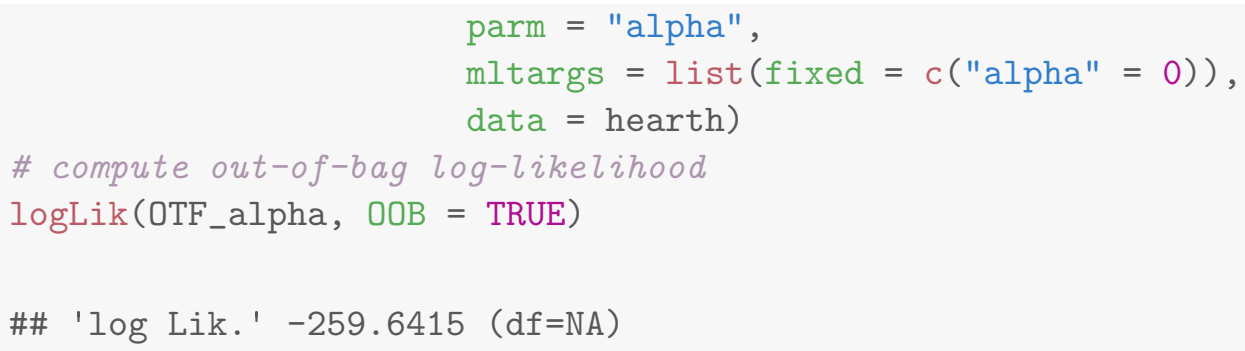

\section{Funding}

This project received funding from the Horizon 2020 Research and Innovation Programme of the European Union under grant agreement number 681094, and is supported by the Swiss State Secretariat for Education, Research and Innovation (SERI) under contract number 15.0137. Torsten Hothorn received funding from the Swiss National Science Foundation under grant number 200021_184603.

\section{References}

Agresti, A. (2002): Categorical Data Analysis, Hoboken, New Jersey, U.S.A.: John Wiley $\&$ Sons, 2nd edition.

Agresti, A. (2010): Analysis of Ordinal Categorical Data, volume 656, Hoboken: John Wiley \& Sons.

Atassi, N., J. Berry, A. Shui, N. Zach, A. Sherman, E. Sinani, J. Walker, I. Katsovskiy, D. Schoenfeld, M. Cudkowicz, et al. (2014): "The PRO-ACT database: Design, initial analyses, and predictive features," Neurology, 83, 1719-1725.

Athey, S., J. Tibshirani, and S. Wager (2019): "Generalized random forests," The Annals of Statistics, 47, 1148-1178.

Beaulieu-Jones, B. K., C. S. Greene, and the Pooled Resource Open-Access ALS Clinical Trials (2016): "Semi-supervised learning of the electronic health record for phenotype stratification," Journal of Biomedical Informatics, 64, 168-178.

Breiman, L., J. H. Friedman, R. A. Olshen, and C. J. Stone (1984): Classification and Regression Trees, California: Wadsworth.

Brooks, B. R., M. Sanjak, S. Ringel, J. England, J. Brinkmann, A. Pestronk, J. Florence, H. Mitsumoto, K. Szirony, and J. Wittes (1996): "The amyotrophic lateral sclerosis functional rating scale: Assessment of activities of daily living in patients with amyotrophic lateral sclerosis," Archives of Neurology, 53, 141-147.

Cedarbaum, J. M., N. Stambler, E. Malta, C. Fuller, D. Hilt, B. Thurmond, and A. Nakanishi (1999): "The ALSFRS-R: A revised ALS functional rating scale that incorporates assessments of respiratory function," Journal of the Neurological Sciences, 169, 13-21. 
Chiò, A., G. Logroscino, O. Hardiman, R. Swingler, D. Mitchell, E. Beghi, and Traynor BG on behalf of the Eurals Consortium (2009): "Prognostic factors in ALS: A critical review," Amyotrophic Lateral Sclerosis, 10, 310-323.

Cohen, J. (1968): "Weighted kappa: Nominal scale agreement provision for scaled disagreement or partial credit." Psychological Bulletin, 70, 213.

Doksum, K. A. and M. Gasko (1990): "On a correspondence between models in binary regression analysis and in survival analysis," International Statistical Review, 58, $243-$ 252.

Friedman, J. H. (1991): "Multivariate adaptive regression splines," The Annals of Statistics, $19,1-67$.

Fujimura-Kiyono, C., F. Kimura, S. Ishida, H. Nakajima, T. Hosokawa, M. Sugino, and T. Hanafusa (2011): "Onset and spreading patterns of lower motor neuron involvements predict survival in sporadic amyotrophic lateral sclerosis," Journal of Neurology, Neurosurgery \& Psychiatry, 82, 1244-1249.

Hemingway, H., P. Croft, P. Perel, J. A. Hayden, K. Abrams, A. Timmis, A. Briggs, R. Udumyan, K. G. M. Moons, E. W. Steyerberg, I. Roberts, S. Schroter, D. G. Altman, and R. D. Riley (2013): "Prognosis research strategy (progress) 1: A framework for researching clinical outcomes," The BMJ, 346.

Hingorani, A. D., D. A. v. d. Windt, R. D. Riley, K. Abrams, K. G. M. Moons, E. W. Steyerberg, S. Schroter, W. Sauerbrei, D. G. Altman, and H. Hemingway (2013): "Prognosis research strategy (progress) 4: Stratified medicine research," The BMJ, 346.

Hornung, R. (2019a): “Ordinal forests," Journal of Classification.

Hornung, R. (2019b): ordinalForest: Ordinal Forests: Prediction and Variable Ranking with Ordinal Target Variables, URL https://CRAN.R-project.org/package= ordinalForest, $\mathrm{r}$ package version 2.3-1.

Hothorn, T. (2019a): TH.data: TH's Data Archive, URL https://CRAN.R-project. org/package=TH . data, $\mathrm{r}$ package version 1.0-10.

Hothorn, T. (2019b): trtf: Transformation Trees and Forests, URL https://CRAN. $R$-project.org/package=trtf, $r$ package version 0.3-6.

Hothorn, T. (2020): "Most likely transformations: The mlt package," Journal of Statistical Software, 92, 1-68.

Hothorn, T., K. Hornik, C. Strobl, and A. Zeileis (2019): party: A Laboratory for Recursive Partytioning, URL https://CRAN.R-project.org/package=party, $r$ package version 1.3-3.

Hothorn, T., K. Hornik, and A. Zeileis (2006): "Unbiased recursive partitioning: A conditional inference framework," Journal of Computational and Graphical Statistics, 15, 651-674.

Hothorn, T. and H. H. Jung (2014): "RandomForest4Life: A random forest for predicting ALS disease progression," Amyotrophic Lateral Sclerosis and Frontotemporal Degeneration, 15, 444-452.

Hothorn, T., B. Lausen, A. Benner, and M. Radespiel-Tröger (2004): "Bagging survival trees," Statistics in Medicine, 23, 77-91. 
Hothorn, T., L. Möst, and P. Bühlmann (2018): "Most likely transformations," Scandinavian Journal of Statistics, 45, 110-134.

Hothorn, T. and A. Zeileis (2015): "partykit: A modular toolkit for recursive partytioning in R," Journal of Machine Learning Research, 16, 3905-3909, URL http://jmlr .org/ papers/v16/hothorn15a.html.

Hothorn, T. and A. Zeileis (2017): "Transformation forests," Technical report, arXiv 1701.02110, v2, URL https://arxiv.org/abs/1701.02110.

Kimura, F., C. Fujimura, S. Ishida, H. Nakajima, D. Furutama, H. Uehara, K. Shinoda, M. Sugino, and T. Hanafusa (2006): "Progression rate of ALSFRS-R at time of diagnosis predicts survival time in ALS," Neurology, 66, 265-267.

Korepanova, N., H. Seibold, V. Steffen, and T. Hothorn (2019): "Survival forests under test: Impact of the proportional hazards assumption on prognostic and predictive forests for ALS survival," Statistical Methods in Medical Research.

Küffner, R., N. Zach, R. Norel, J. Hawe, D. Schoenfeld, L. Wang, G. Li, L. Fang, L. Mackey, O. Hardiman, M. Cudkowicz, A. Sherman, G. Ertaylan, M. Grosse-Wentrup, T. Hothorn, J. van Ligtenberg, J. H. Macke, T. Meyer, B. Schölkopf, L. Tran, R. Vaughan, G. Stolovitzky, and M. L. Leitner (2015): "Crowdsourced analysis of clinical trial data to predict amyotrophic lateral sclerosis progression," Nature Biotechnology, 33, 51-57.

Lin, Y. and Y. Jeon (2006): "Random forests and adaptive nearest neighbors," Journal of the American Statistical Association, 101, 578-590.

Mandrioli, J., E. Rosi, N. Fini, A. Fasano, S. Raggi, A. L. Fantuzzi, and G. Bedogni (2017): "Changes in routine laboratory tests and survival in Amyotrophic Lateral Sclerosis," Neurological Sciences, 38, 2177-2182.

McCullagh, P. (1980): "Regression Models for Ordinal Data," Journal of the Royal Statistical Society: Series B (Methodological), 42, 109-127.

Meinshausen, N. (2006): "Quantile regression forests," Journal of Machine Learning Research, 7, 983-999, URL http://jmlr.org/papers/v7/meinshausen06a.html.

Moons, K. G. M., P. Royston, Y. Vergouwe, D. E. Grobbee, and D. G. Altman (2009): "Prognosis and prognostic research: What, why, and how?" The BMJ, 338.

Ong, M.-L., P. F. Tan, and J. D. Holbrook (2017): "Predicting functional decline and survival in Amyotrophic Lateral Sclerosis," PLoS ONE, 12, e0174925.

Peterson, R. L., D. M. Vock, J. H. Powers, S. Emery, E. F. Cruz, S. Hunsberger, M. K. Jain, S. Pett, J. D. Neaton, and I. F.-I. S. Group (2017): "Analysis of an ordinal endpoint for use in evaluating treatments for severe influenza requiring hospitalization," Clinical Trials, 14, 264-276.

Pfohl, S. R., R. B. Kim, G. S. Coan, and C. S. Mitchell (2018): "Unraveling the complexity of Amyotrophic Lateral Sclerosis survival prediction," Frontiers in Neuroinformatics, 12, 12.

R Core Team (2020): $R$ : A Language and Environment for Statistical Computing, R Foundation for Statistical Computing, Vienna, Austria, URL https://www .R-project . org/. Riley, R. D., J. A. Hayden, E. W. Steyerberg, K. G. Moons, K. Abrams, P. A. Kyzas, N. Malats, A. Briggs, S. Schroter, D. G. Altman, et al. (2013): "Prognosis research 
strategy (progress) 2: Prognostic factor research,” PLoS Medicine, 10, e1001380.

Roozenbeek, B., H. F. Lingsma, P. Perel, P. Edwards, I. Roberts, G. D. Murray, A. I. Maas, and E. W. Steyerberg (2011): "The added value of ordinal analysis in clinical trials: An example in traumatic brain injury," Critical Care, 15, R127.

Royston, P., K. G. Moons, D. G. Altman, and Y. Vergouwe (2009): "Prognosis and prognostic research: Developing a prognostic model," The BMJ, 338, b604.

Schlosser, L., T. Hothorn, R. Stauffer, and A. Zeileis (2019): "Distributional regression forests for probabilistic precipitation forecasting in complex terrain," The Annals of Applied Statistics, 13, 1564-1589.

Schmid, M., T. Hothorn, K. O. Maloney, D. E. Weller, and S. Potapov (2011): "Geoadditive regression modeling of stream biological condition," Environmental and Ecological Statistics, 18, 709-733.

Seibold, H., A. Zeileis, and T. Hothorn (2018): "Individual treatment effect prediction for Amyotrophic Lateral Sclerosis patients," Statistical Methods in Medical Research, 27, 3104-3125.

Steyerberg, E. W., K. G. Moons, D. A. van der Windt, J. A. Hayden, P. Perel, S. Schroter, R. D. Riley, H. Hemingway, D. G. Altman, P. Group, et al. (2013): "Prognosis research strategy (progress) 3: Prognostic model research," PLoS Medicine, 10, e1001381.

Tanadini, L. G., J. D. Steeves, A. Curt, and T. Hothorn (2016): "Autoregressive transitional ordinal model to test for treatment effect in neurological trials with complex endpoints," BMC Medical Research Methodology, 16, 149.

Tutz, G. (2011): Regression for Categorical Data, volume 34, New York: Cambridge University Press.

Whegang, S. Y., L. K. Basco, H. Gwét, and J.-C. Thalabard (2010): "Analysis of an ordinal outcome in a multicentric randomized controlled trial: Application to a 3-arm antimalarial drug trial in cameroon," BMC Medical Research Methodology, 10, 58.

Winell, H. and J. Lindbäck (2018): "A general score-independent test for order-restricted inference," Statistics in Medicine, 37, 3078-3090.

Zoccolella, S., E. Beghi, G. Palagano, A. Fraddosio, V. Guerra, V. Samarelli, V. Lepore, I. L. Simone, P. Lamberti, L. Serlenga, and Logroscino G for the SLAP Registry (2008): "Analysis of survival and prognostic factors in amyotrophic lateral sclerosis: A population based study," Journal of Neurology, Neurosurgery \& Psychiatry, 79, 33-37. 


\section{A Additional Results of the Empirical Evaluation}

\section{A.1 True Log-Likelihood vs. Random Forest Based Log-Likelihoods}

\section{A.1.1 Log-Likelihood Differences}

Same as Figure 1, but based on forests of 2000 trees instead of aggregating over 250 trees only.

\section{A.1.2 Log-Likelihood}

Figures 1 and 4 present log-likelihood differences; for the same simulations, the raw loglikelihoods (without centering with respect to the log-likelihood of the true data-generating process) are given in Figures 5 and 6 with 250 and 2000 trees, respectively.

\section{A.2 Kullback-Leibler Divergence}

The out-of-sample log-likelihood assesses the quality of a predictive distribution obtained from any of the variants of Random Forests for Ordinal Regression in light of an independent validation sample, where the ordinal response was sampled from the underlying data-generating process. This comparison might be unfair because Ordinal Forests were designed to focus on predictions of the outcome categories, not predictive distributions. Hence, it was suggested by an external referee to use an alternative performance measure which allows to assess the quality of a random forest based on the predicted outcome categories.

Let $p\left(c_{k} \mid x_{l}\right)=\mathbb{P}\left(Y_{l}=c_{k} \mid x_{l}\right)$ denote the true conditional probability density of the $\imath$ th observation in the validation sample over the $K$ possible categories $c_{1}, \ldots, c_{K}$, which is known in case of our simulation study for a configuration $x_{l}$ of the explanatory variables. With $\hat{p}\left(c_{k} \mid x_{l}\right)=\hat{\mathbb{P}}\left(Y_{l}=c_{k} \mid x_{l}\right)$ we denote the predictive conditional probability density obtained from any of the random forest variants studied in this paper.

The Kullback-Leibler divergence evaluated for the $\tilde{N}$ validation samples

$$
\mathrm{KL}=\sum_{l=N+1}^{N+\tilde{N}} \sum_{k=1}^{K} p\left(c_{k} \mid x_{l}\right) \log \left(\frac{p\left(c_{k} \mid x_{l}\right)}{\hat{p}\left(c_{k} \mid x_{l}\right)}\right)
$$

allows a direct comparison of true and estimated conditional probability densities. The results for each of the eight simulation scenarios for 250 and 2000 trees are given in Figures 7 and 8. The same conclusions as drawn in the main text hold, i.e. $\operatorname{OTF}(\alpha)$ performed best in the proportional odds scenario and $\mathrm{OTF}(\vartheta)$ seemed to be able to detect deviations from this model assumption. Ordinal Forests performed less well.

As a special case, the predicted Dirac distribution putting mass one on category $k^{\prime}$ of the $K$ categories, is denoted by $d_{k^{\prime}}\left(c_{k} \mid x_{l}\right)=I\left(k=k^{\prime}\right)$. This distribution represents the 


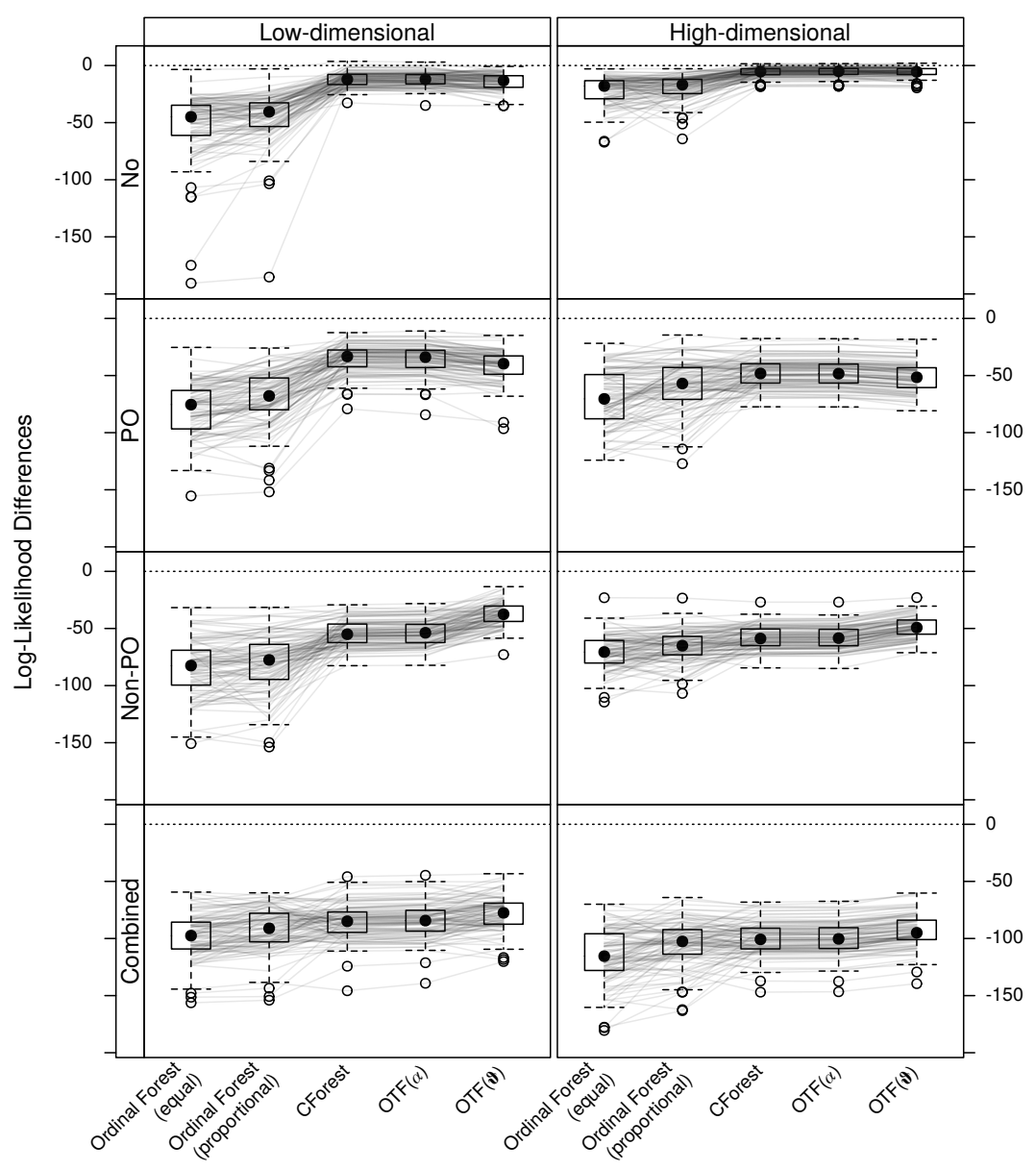

Figure 4 


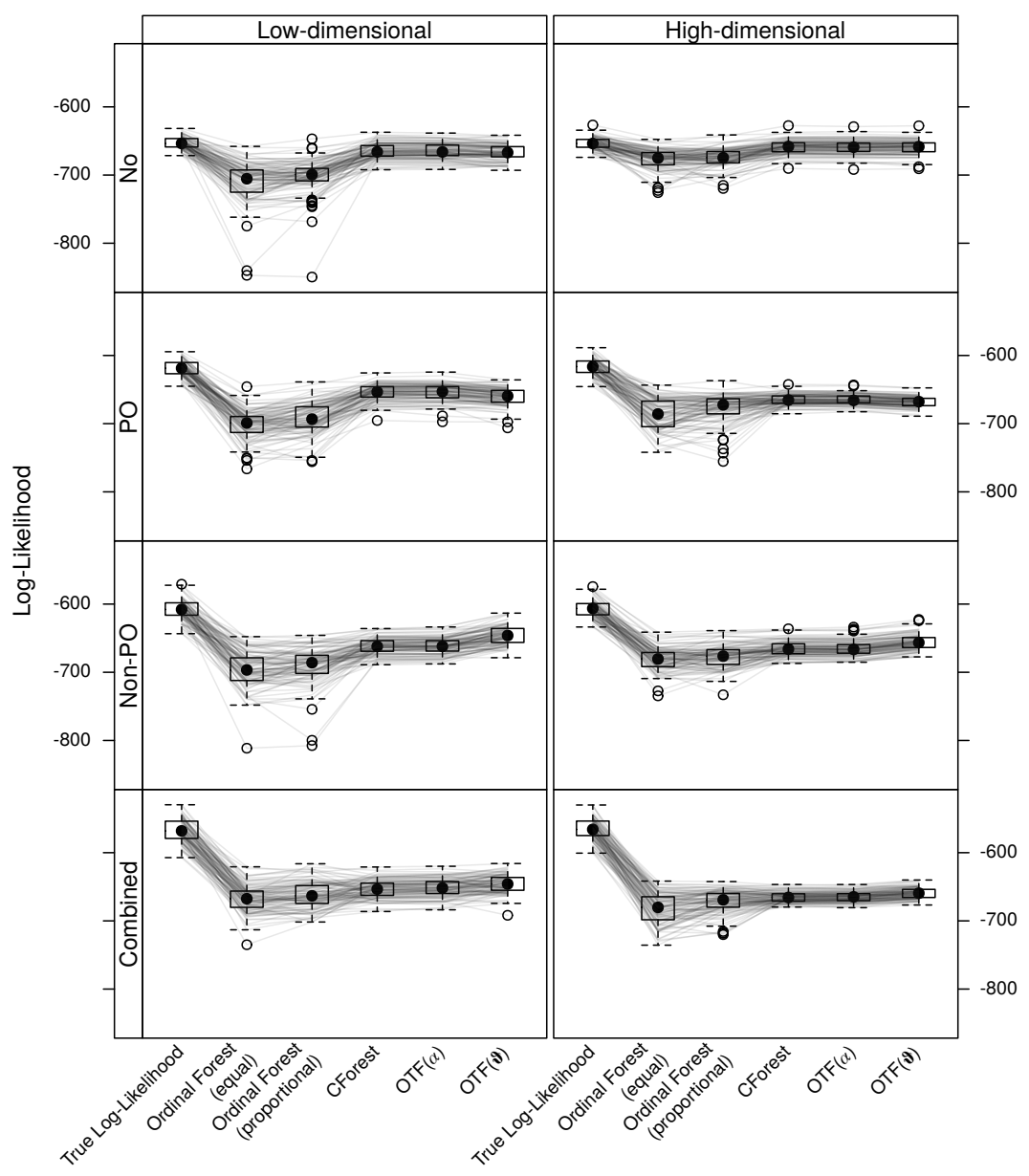

Figure 5 


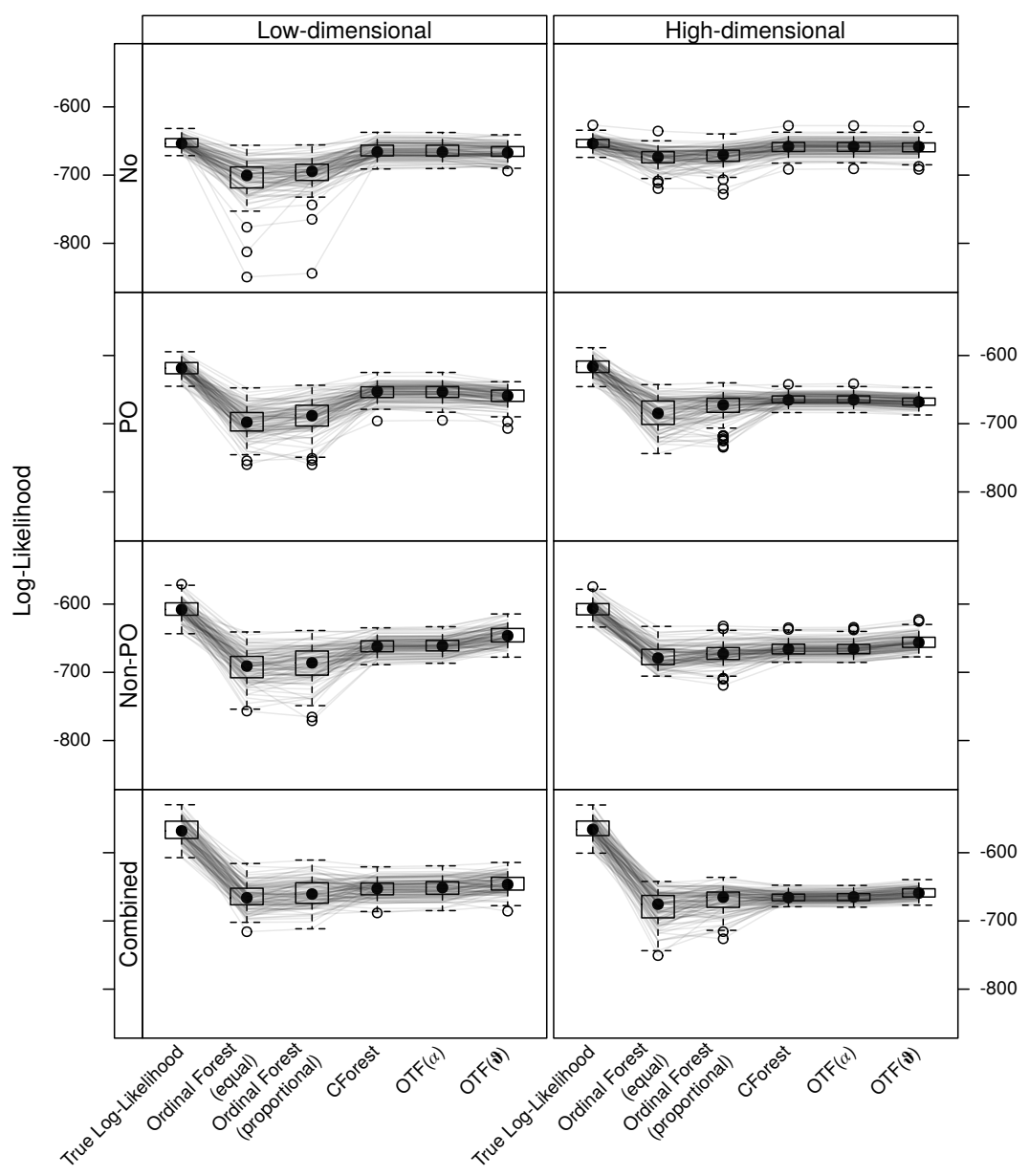

Figure 6 


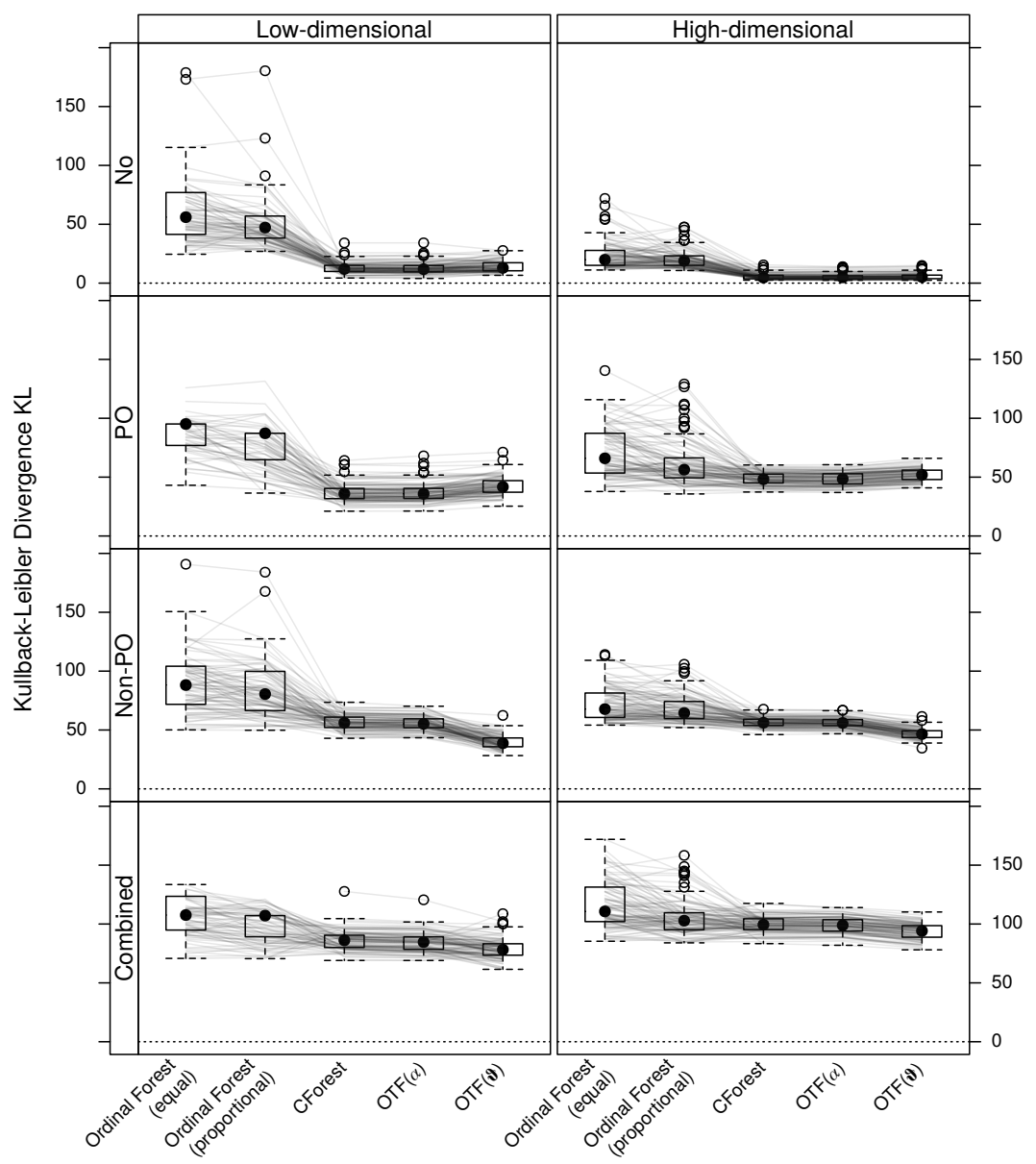

Figure 7 


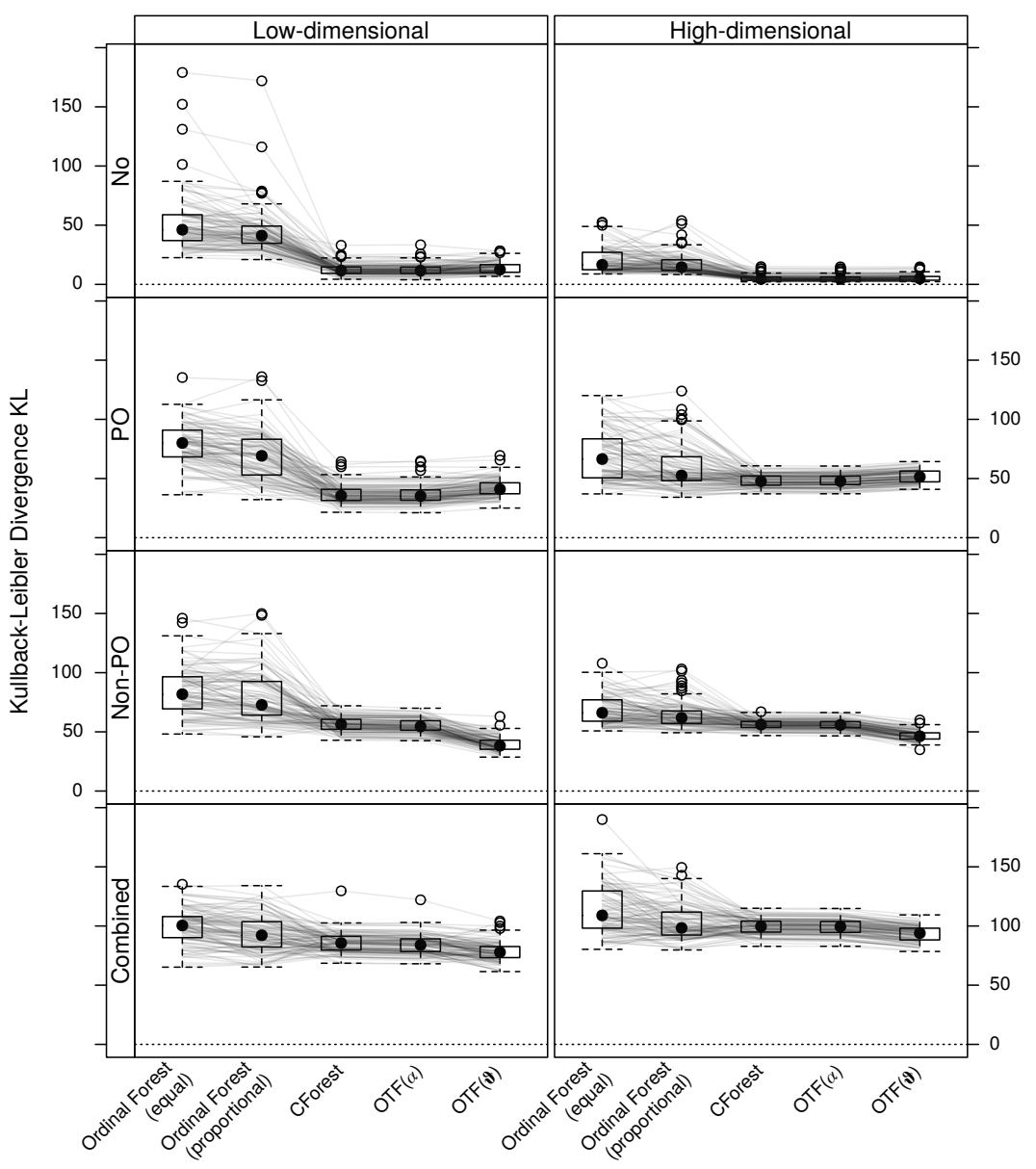

Figure 8 
empirical density of an observation $Y_{l}=c_{k^{\prime}}$ from the true data-generating process or a point prediction $\hat{Y}_{l}=c_{k^{\prime}}$.

When replacing the true conditional probability density $p$ with a realisation $Y_{l}$ from this density, i.e. putting empirical mass one on an observation $Y_{l}$ with explanatory variables $x_{l}$, the Kullback-Leibler divergence is equivalent to the negative log-likelihood

$$
\mathrm{KL}_{1}=\sum_{l=N+1}^{N+\tilde{N}} \sum_{k=1}^{K} d_{Y_{l}}\left(c_{k} \mid x_{l}\right) \log \left(\frac{d_{Y_{l}}\left(c_{k} \mid x_{l}\right)}{\hat{p}\left(c_{k} \mid x_{l}\right)}\right)=\sum_{l=N+1}^{N+\tilde{N}}-\log \left(\hat{p}\left(Y_{l} \mid x_{l}\right)\right)
$$

For the sake of completeness, Figures 9 and 10 are printed in addition to Figures 5 and 6 , again for 250 and 2000 trees.

Finally, we can compare a point prediction $\hat{Y}_{l}$ (the default output of predict . ord() from ordinalForest (Hornung, 2019a)) with the true probability density $p$ by using a Dirac distribution putting mass one on $\hat{Y}_{l}$ in

$$
\mathrm{KL}_{2}=\sum_{l=N+1}^{N+\tilde{N}} \sum_{k=1}^{K} d_{\hat{Y}_{l}}\left(c_{k} \mid x_{l}\right) \log \left(\frac{d_{\hat{Y}_{l}}\left(c_{k} \mid x_{l}\right)}{p\left(c_{k} \mid x_{l}\right)}\right)=\sum_{l=N+1}^{N+\tilde{N}}-\log \left(p\left(\hat{Y}_{l} \mid x_{l}\right)\right.
$$

This measure provides a fair comparison with Ordinal Forests, because no additional effort to obtain a predictive distribution is necessary in order to compute this performance measure. The results were, however, in line with the findings from the other two KullbackLeibler divergence metrics and the log-likelihood evaluations (see Figures 11 and 12). 


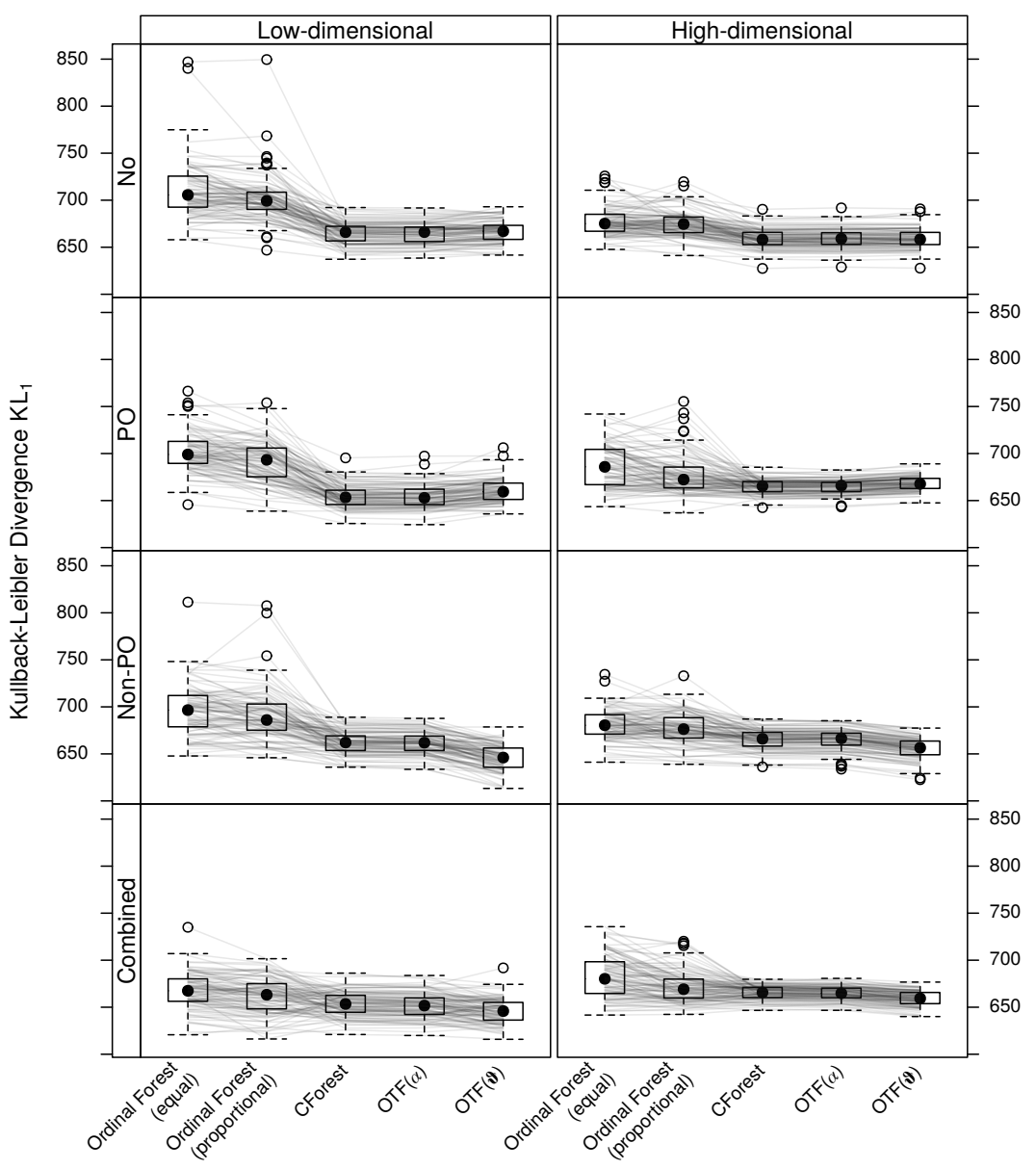

Figure 9 


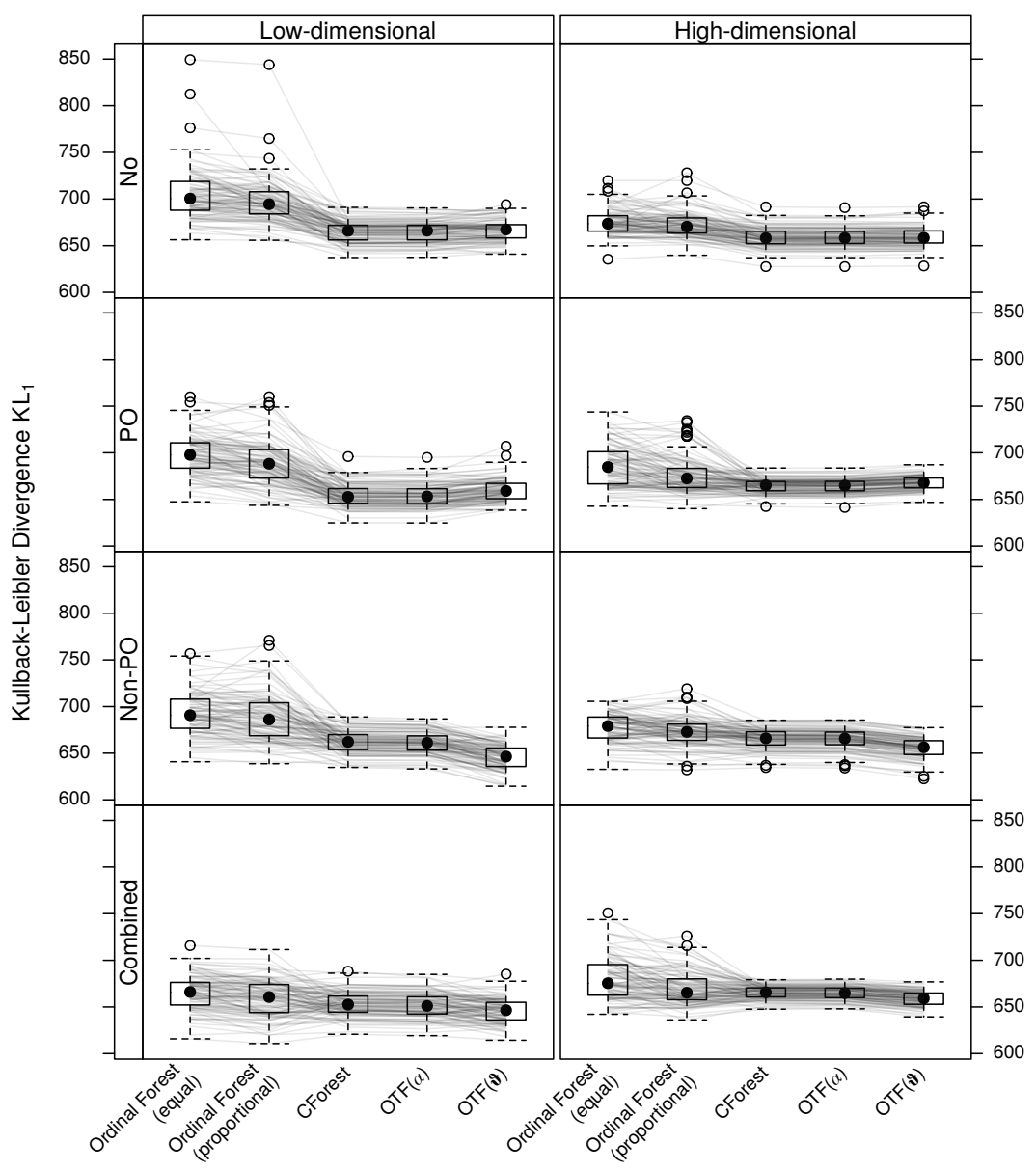

Figure 10 


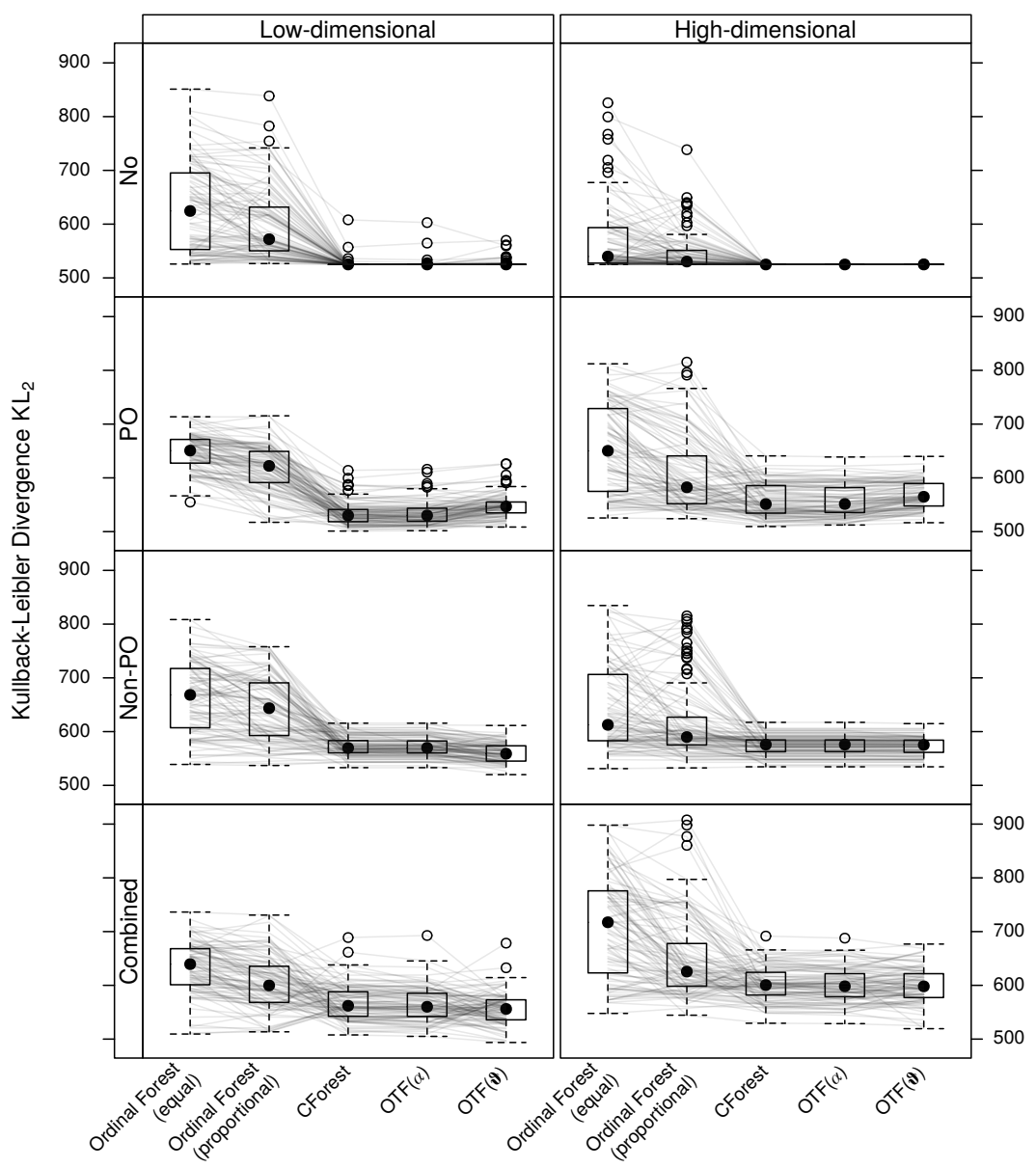

Figure 11 


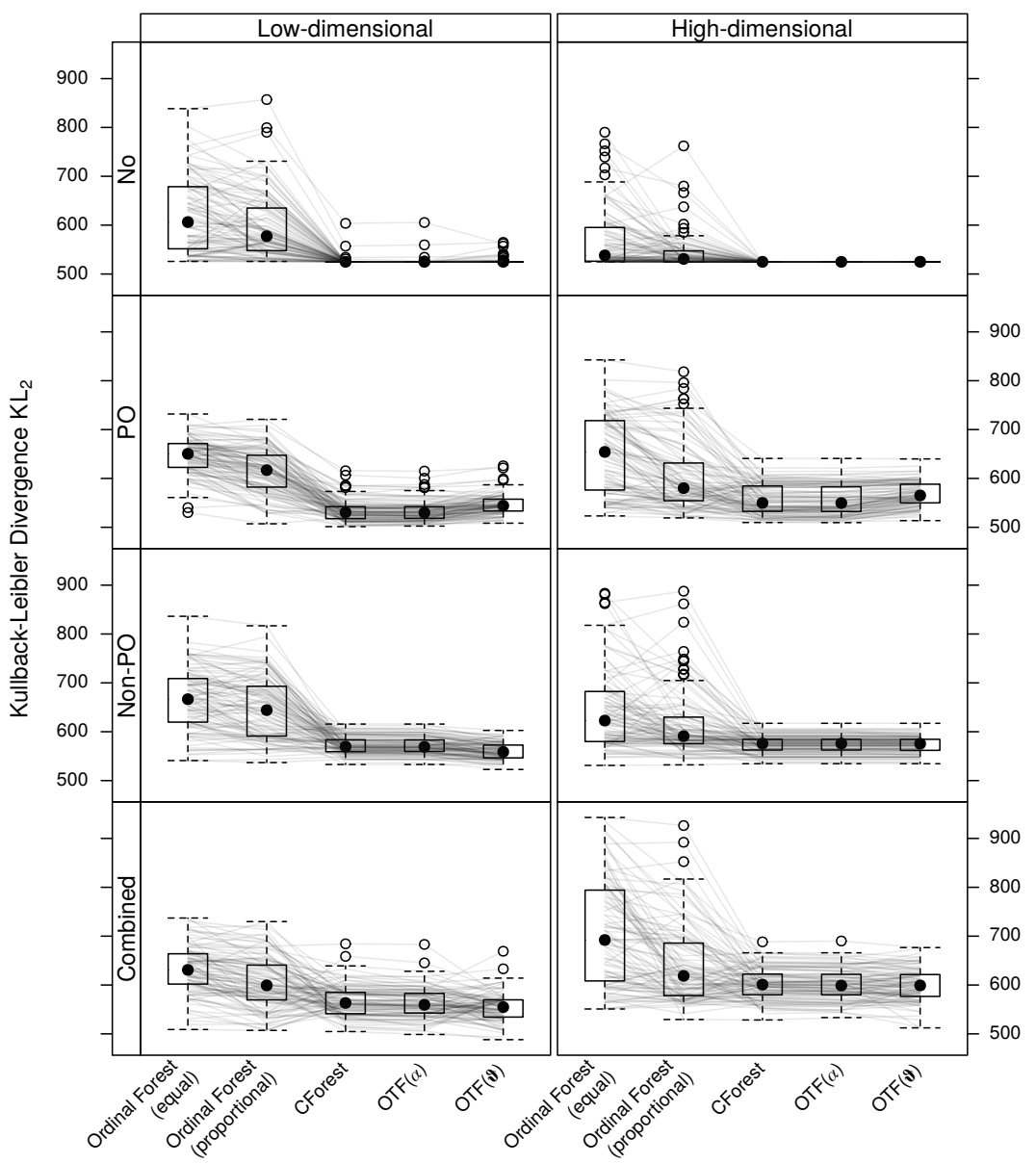

Figure 12 